\title{
Dynamic simulation of seat suspension system with virtual prototyping technology
}

\author{
Thanh Danh LE* and Phuong T NGUYEN ** \\ * Faculty of Mechanical Engineering, Ho Chi Minh City University of Technology \\ 268 Ly Thuong Kiet Street-District10-HoChiMinh City, Viet Nam \\ E-mail: le_thanh_danh@hotmail.com \\ ${ }^{* *}$ Ho Chi Minh City University of Food Industry \\ 140 Le Trong Tan Street-Tan Phu District-HoChiMinh City, Viet Nam
}

Received: 27 June 2017; Revised: 18 August 2017; Accepted: 19 September 2017

\begin{abstract}
This paper will describe and simulate the dynamic response of an innovative suspension system for vehicle seat. In which, the isolated object is supported by a wedge mechanism which comprises a horizontal spring, a roller and a wedge. Besides, the dynamic stiffness of system is corrected by an auxiliary mechanism (AM) including a roller (follower) which always contacts the circular surface of the cam via another horizontal spring. Hence, this seat suspension prototype not only broad the excitation frequency region but also remains the load bearing capacity and is named by "model with AM". The dynamic stiffness-configurative parameter relationship is presented. Then, a virtual mechanical prototyping model of the system is created by using SOLIDWORKS software. Next, by applying dynamic analysis in COSMOSMotion module of the Solidworks product family, the real behaviour of system is tested and evaluated without using the traditional build-and-test method. With this way, it can increase the quality of product by reducing manufacturing cost and error. The simulation results show that the model with AM outperforms the linear counterpart. Besides, these results furnish a useful insight for the design and manufacture of the seat suspension system for vehicle.
\end{abstract}

Keywords : Seat suspension, Low dynamic stiffness, Low frequency isolation, Virtual model

\section{Introduction}

As known, one of main causes that which induce many damages of machinery, equipment is undesirable vibrations. Particularly, vibrations that occur in low frequency regions $(<5 \mathrm{~Hz})$ are almost harmful and dangerous to human health and activity researched by (Paddan, 2002). Thus, suspensions are widely used to lengthen the service life of the machinery, equipment as well as to provide more comfortable and safe condition for human in vehicle. Commonly, a linear system (conventional system), which is widely used for isolating unwanted vibrations, consists of a linear stiffness spring, $K$, in parallel to a damper, $C$ in (Thorby, 2008) and (Graham Kelly, 2002). The performance of vibration isolation can be only effective when the excitation frequency is greater than $\sqrt{2}$ times the natural frequency of the system. This limitation confines a conventional system to attenuate vibration in low frequency band. One of strategies for extending isolation ability to low frequency domain is to reduce the stiffness of the system. However, a reduction in stiffness results in a low load bearing capacity and an excessive static deformation of the system. This could cause many difficulties for designing a traditional system. Currently, there are many researchers, engineers and scholars who have suggested solutions to reduce the resonance frequency such as a highly deformed elastic structure working as a nonlinear spring is used to support a static load as presented by (Chnin et al., 2005), (Plaut et al., 2008), (Virgin et al., 2003) and (Winterflood et al., 2002) or another structure that is also based on the elastic model with extreme geometric nonlinearity is studied by (Santillan et al., 2005) and (Virgin et al., 2008). The purpose of elastic structures is to reduce the resonance frequency. However, these structures easily lose stability when the stiffness of the elastic elements is low.

In order to overcome the dichotomy between load bearing capacity and reduction in stiffness, in recent years, quasi-zero stiffness (QZS) vibration isolation systems have been studied and developed by (Carrella et al, 2007, 2008 and 2012), (Hao et al., 2015) and (Kovacic et al, 2008). These systems are also referred to as high-static low-dynamic 
stiffness (HSLD) vibration isolation systems. Due to the negative and positive elements connected in parallel, the HSLD obtains the low dynamic stiffness around the static equilibrium position but still remains the load bearing capacity. Hence, this isolation way is available to achieve a low isolation frequency band. Currently, the QZS vibration isolation systems have been studied in great depth and widely applied in many fields such as the suspensions of the ground vehicle and seat, nanotech and the space shuttle. A spring with negative stiffness is designed by (Lee et al., 2007) to improve vehicle driver vibration isolation seat in low excitation frequency region. This design presents an approach, based on the consistent theory of thin shells, for designing compact springs in terms of their compatibility with the room available for packaging the vehicle suspensions and simultaneous extension of the height control region where fundamental frequencies are kept minimal. In addition, some active isolation systems have been also studied and applied in practice. Such as, Nakamura, 2013 studied and developed the Smith predictor to improve the isolation performance of the pneumatic active anti-vibration system or Shirani et al, 2013 designed the flow disturbance compensation based on the off-line estimated signal for a pneumatic isolation table.

Drawn by the QZS isolation approach, we will propose a seat suspension system for vehicle drive. For simplicity, the weight, elasticity and stiffness of the seat cushion and the damping of the components inside of a human body such as the spine, are all ignored. This paper only focuses the dynamic simulation of the seat isolation system when the system achieves the desirable static equilibrium position. In this system, an auxiliary mechanism is used to correct the dynamic stiffness of seat suspension model. In addition, the isolated object is supported by another part called the load bearing mechanism (LBM). To ensure the actual performance of the system, the virtual prototyping technology via SOLIDWORKS software is employed to simulate the dynamic behaviour of the proposed model. This paper is organized as follows. Seat suspension model is described in section 2. Next, the dynamic stiffness of the system is analyzed in section 3. The virtual model is created in section 4 . The simulation results are presented in section 5. Finally, some conclusions are drawn in section 6.

\section{Seat suspension system}

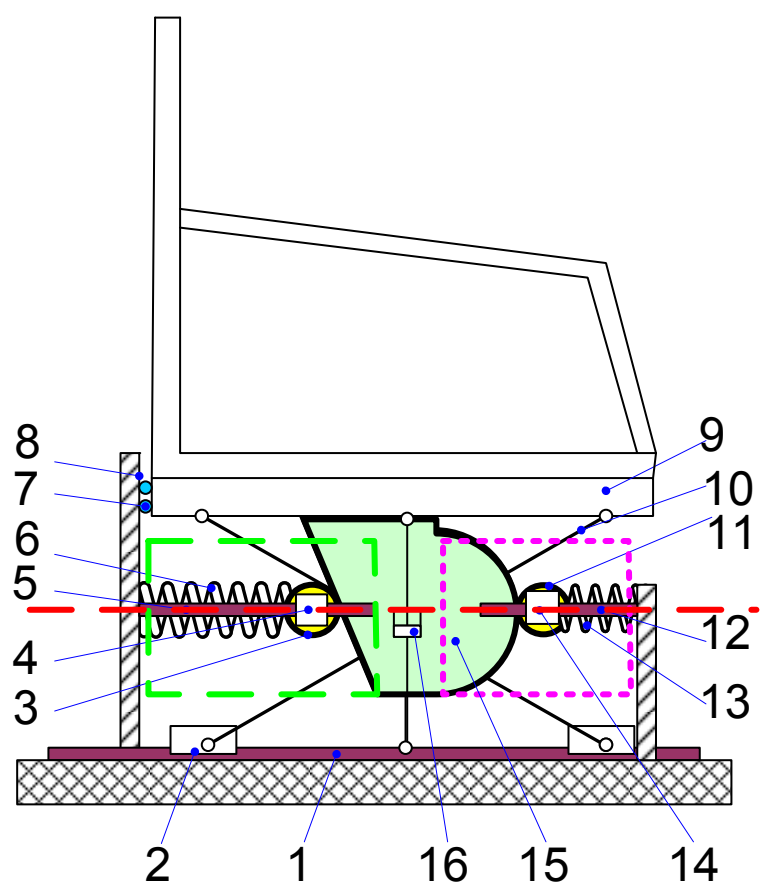

Fig. 1. Schematic diagram of seat suspension system: 1, 5 and 12 - linear rail, 2, 4 and 14 - linear slider, 6 and 13 - horizontal spring,

7 - roller, 8- guide, 9 - seat frame, 10 - rod, 3 and 11 - follower with radius r, 15 -wedge-cam, and 16 - damper.

The scissor-like structured seat suspension system with wedge-cam-roller-spring mechanism is designed as shown in Fig. 1. The wedge-cam-roller-spring mechanism consists of a wedge, a semi-circular cam, two rollers (3 and 11) and two springs (6 and 13). The wedge and semi-circular cam are integrated into a part (15) named the wedge-cam. The proposed system is divided into two main portions. The left portion denoted by the green dashed-line box is the load bearing mechanism (LBM) which consists of a horizontal spring (6), a roller (3) and a wedge-cam (15). When the suspension is loaded, the wedge-cam which is fixed the bottom of seat frame (9) compresses the horizontal spring (6) through roller 3 
moving along linear rail (5). The right one is the auxiliary mechanism (AM) plotted by pink dot-line box in which the horizontal spring (13) is always compressed to remain the contact between the circular surface of the wedge-cam (15) and follower (11). The seat is only moved in vertical direction via the roller (7) and guide (8). The position at which the isolated object is only supported by the compressible spring 6 is called the desirable static equilibrium position as displayed by red dashed line. Due to the opposite between the LBM having positive stiffness and the AM acting as negative stiffness element, the seat suspension system can achieve the low resonance frequency and peak but still remains the load bearing capacity. By this way, the proposed system can remove major disadvantages of traditional isolation seats.

\section{Dynamic stiffness of the system}

In order to analyze to isolation effectiveness of the designed system, the relationship between the dynamic stiffness and configurative parameters of the system is studied and discussed. Considering the isolated object $(m)$ is displaced downward an amount, $y$, from the initial position $O_{1}$ to $O^{\prime}{ }_{1}$. The results is that the left roller is displaced from the initial position $\mathrm{O}_{2}$ to $\mathrm{O}_{2}{ }_{2}$ and the right roller is moved from the initial position $\mathrm{O}_{3}$ to $\mathrm{O}_{3}{ }_{3}$. Here, the black dashed line denoted the initial position of the seat suspension system at which the two springs are unloaded. The displacement relation between two rollers and the wedge-cam is presented in Fig. 2.

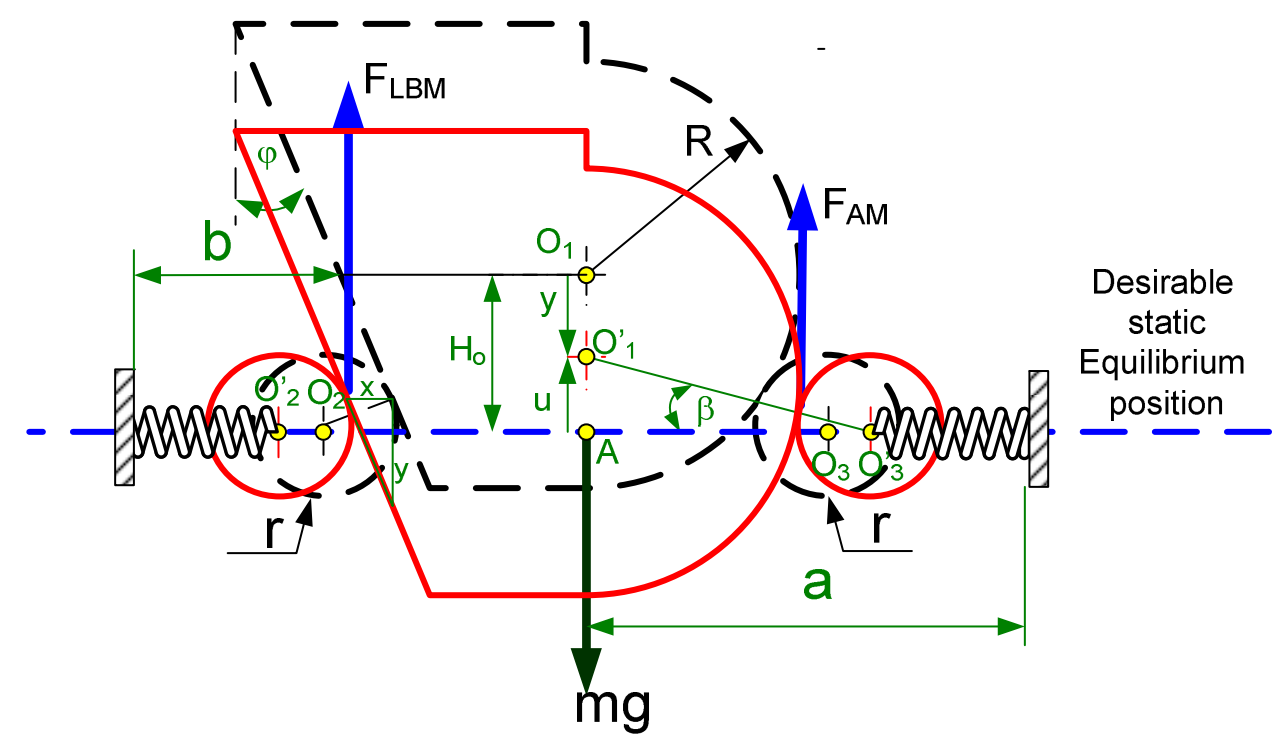

Fig. 2: Force acting on the wedge-cam.

The deformations of the left $\left(\Delta L_{1}\right)$ and right $\left(\Delta L_{2}\right)$ spring are determined as:

$$
\begin{aligned}
& \Delta L_{1}=x=y \tan \varphi \\
& \Delta L_{2}=\sqrt{(R+r)^{2}-\left(H_{o}-y\right)^{2}}-\sqrt{(R+r)^{2}-H_{o}^{2}}
\end{aligned}
$$

where, $H_{o}=m g /\left(K_{l} \tan ^{2} \varphi\right)$ is the static deformation of the system in vertical direction as shown in Fig. 2 . $K_{l}$ is the stiffness of the left spring, $m$ is the weight of the isolated object and $\varphi$ is the inclined angle of the wedge-cam.

If letting $u=H_{o}-y$, the Eq.(1) is rewritten as following:

$$
\begin{aligned}
& \Delta L_{1}=\left(H_{o}-u\right) \tan \varphi \\
& \Delta L_{2}=\sqrt{(R+r)^{2}-u^{2}}-\sqrt{(R+r)^{2}-H_{o}{ }^{2}}
\end{aligned}
$$

The force of the LBM $\left(\mathrm{F}_{L B M}\right)$ and the AM $\left(\mathrm{F}_{A M}\right)$ acting on the wedge-cam in the vertical direction are determined by Eq.(4-5):

$$
F_{L B M}=K_{1} \Delta L_{1} \tan \varphi
$$




$$
F_{A M}=K_{2} \Delta L_{2} \tan \beta
$$

where $K_{2}$ is the stiffness of the right spring. The angle of $\beta$ is determined by Eq.(6).

$$
\tan \beta=\frac{u}{\sqrt{(R+r)^{2}-u^{2}}}
$$

From Eq. (2-6), the vertical restoring force - displacement relationship is expressed as following:

$$
F=K_{1}\left(H_{o}-u\right) \tan ^{2} \varphi+K_{2}\left(\sqrt{(R+r)^{2}-u^{2}}-\sqrt{(R+r)^{2}-H_{o}^{2}}\right) \frac{u}{\sqrt{(R+r)^{2}-u^{2}}}
$$

The dynamic stiffness of the system can be obtained by differentiating the Eq. (7) with respective displacement $u$ as following:

$$
K=K_{1} \tan ^{2} \varphi-K_{2}+K_{2} \sqrt{(R+r)^{2}-H_{o}^{2}}\left(\frac{u^{2}}{\sqrt{\left((R+r)^{2}-u^{2}\right)^{3}}}+\frac{1}{\sqrt{(R+r)^{2}-u^{2}}}\right)
$$

It is convenient for introducing non-dimensional form:

$$
\begin{aligned}
& \hat{K}=\frac{K}{K_{1}} ; \alpha=\frac{K_{2}}{K_{1}} ; \hat{u}=\frac{u}{\sqrt{(R+r)^{2}-H_{o}^{2}}} ; \\
& \hat{R}=\frac{R}{\sqrt{(R+r)^{2}-H_{o}^{2}}} ; \hat{r}=\frac{r}{\sqrt{(R+r)^{2}-H_{o}^{2}}}
\end{aligned}
$$

By letting $\hat{\mathbb{R}}=\hat{R}+\hat{r}$, Eq. (7) is rewritten as following:

$$
\hat{K}=\tan ^{2} \varphi-\alpha\left(1-\frac{\hat{u}^{2}}{\sqrt{\left(\hat{\mathbb{R}}^{2}-\hat{u}^{2}\right)^{3}}}-\frac{1}{\sqrt{\hat{\mathbb{R}}^{2}-\hat{u}^{2}}}\right)
$$

The dynamic stiffness at the static equilibrium position $\hat{K}_{S E P}$ is determined by Eq. (11).

$$
\hat{K}_{S E P}=\tan ^{2} \varphi-\alpha\left(1-\frac{1}{\hat{\mathbb{R}}}\right)
$$

In addition, according to geometric relation as shown in Fig. 2, the distance $H_{o}$ is also expressed as following:

$$
H_{o}=\sqrt{(R+r)^{2}+\left(a-L_{o 2}\right)^{2}}
$$

where the parameter of $a$ is the horizontal distance from the wall to the center $O_{l}$ as shown in Fig.2, the Eq.(11) is rewritten as following:

$$
\hat{K}_{S E P}=\tan ^{2} \varphi-\alpha\left(1-\frac{\sqrt{(R+r)^{2}-\left((R+r)^{2}+\left(a-L_{o 2}\right)^{2}\right)}}{R+r}\right)
$$

Figure. 3 presents the influence of the inclined angle $(\varphi)$ of the wedge-cam and the spring ratio $(\alpha)$ on the dynamic stiffness $\left(\hat{K}_{S E P}\right)$ at the static equilibrium position when $\hat{\mathbb{R}}=1.37$. This figure reveals that the value of $\hat{K}_{S E P}$ is increased versus the increase of the values of $\alpha$ and $\varphi$. However, it can be seen that if the spring ratio and inclined angle of the wedge-cam are within the pink region, the $\left(\hat{K}_{S E P}\right)$ is negative. This can leads to instability of the suspension system.

In addition the dynamic stiffness characteristic of the system also depends on the displacement of the isolated object as plotted in Fig. 4, here the parameter $\hat{\mathbb{R}}$ is the same as in Fig. 3. The stiffness curve is a concave parabola and the stiffness of the system is increased when the isolated mass moves away from the static equilibrium position. 
Furthermore, it can be observed from Fig. 4(a) that when the value of $\varphi$ is $37^{\circ}$, the opposite of the increasing the dynamic stiffness $\left(\hat{K}_{S E P}\right)$, the slope of the stiffness curve is reduced according to growing the spring ratio $\alpha$. On the other hand, if the spring ratio $\alpha$ is kept at the value of 1.037 , the dynamic stiffness at the static equilibrium position and the slope of the stiffness curve are increased if the inclined angle $\varphi$ of the wedge-cam is increased as shown in Fig. 4(b).

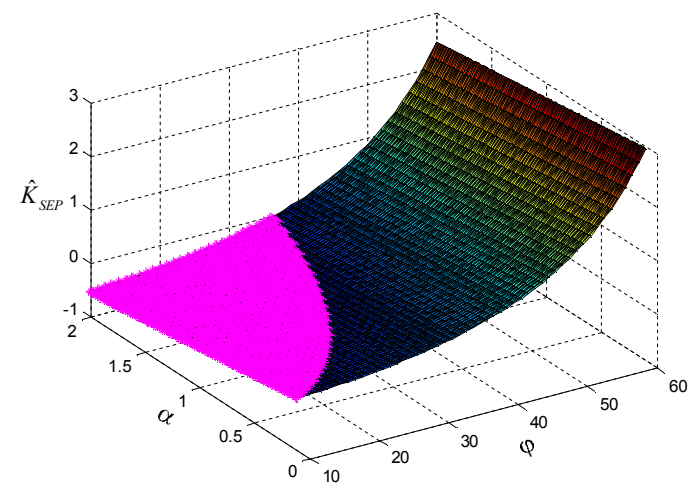

Fig. 3: Influence of the spring ratio $(\alpha)$ and the inclined angle $(\varphi)$ of the cam on the dynamic stiffness value at the static equilibrium position with $\hat{\mathbb{R}}=1.37$.

a.
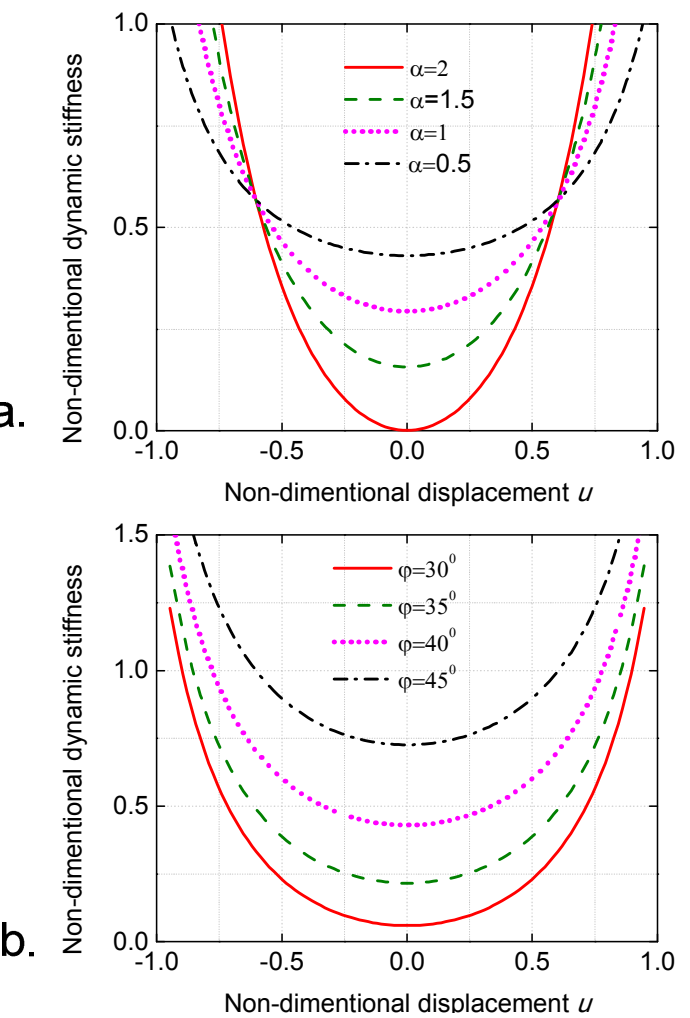

Fig. 4: Plot of dynamic stiffness curve: (a) $\varphi=37^{\circ}$ and $\alpha=0.5$ (Black dashed-dot line); 1 (Pink dot line); 1.5 (Olive dashed line); and 2(Red solid line), (b) $\alpha=1.037$ and $\varphi=45^{\circ}$ (Black dashed-dot line); $40^{\circ}$ (Pink dot line); $35^{\circ}$ (Olive dashed line); and $30^{\circ}$ ( Red solid line).

From Eq. (14) it can be seen that there is a unique relationship between the parameters such that the dynamic stiffness at the static equilibrium position is quasi-zero. As can be seen from Fig. 5(a) that the inclined angle $\varphi$ of the wedge-cam is increased according to the increase of parameter $\hat{\mathbb{R}}$ or the spring ratio $\alpha$. Especially, when the value of $\hat{\mathbb{R}}$ is around the value of one, the value of the inclined angle $\varphi$ is reduced closer to zero degree. In this case, the load bearing capacity of the proposed model is bad. Hence, in order to ensure, the parameter $\hat{\mathbb{R}}$ must be larger than one.

In addition, the slope of the dynamic stiffness curve that which is expressed by the distance $d$ on which the dimensionless dynamic stiffness is always smaller than one is described in Eq. (15). By combinating Eq. (14) and (15) the relationship between the configurative parameters and the distance $d$ is given by condition. (16). It can be observed 
that the distance $d$ is increased according to the increase of the parameter $\hat{\mathbb{R}}$ as shown in Fig. 5(b). Conversely, the value of $d$ will be reduced when there is an increase in the spring ratio $\alpha$. This is also confirmed by numerical solutions of Eq. (10) as shown in Fig. 6.

$$
\begin{aligned}
& \tan ^{2} \varphi=\alpha\left(\frac{\hat{\mathbb{R}}-1}{\hat{\mathbb{R}}}\right) \\
& \hat{d}=2 \sqrt{\left[\hat{\mathbb{R}}^{2}-\sqrt[3]{\left(\frac{\mathbb{R}^{2} \alpha}{1+\alpha-\tan ^{2} \varphi}\right)^{2}}\right]} \\
& \left\{\begin{array}{l}
\hat{\mathbb{R}}>1 \\
\hat{d}=2 \hat{\mathbb{R}} \sqrt{\left[1-\sqrt[3]{\left(\frac{\alpha}{\hat{\mathbb{R}}+\alpha}\right)^{2}}\right]}
\end{array}\right.
\end{aligned}
$$

a.

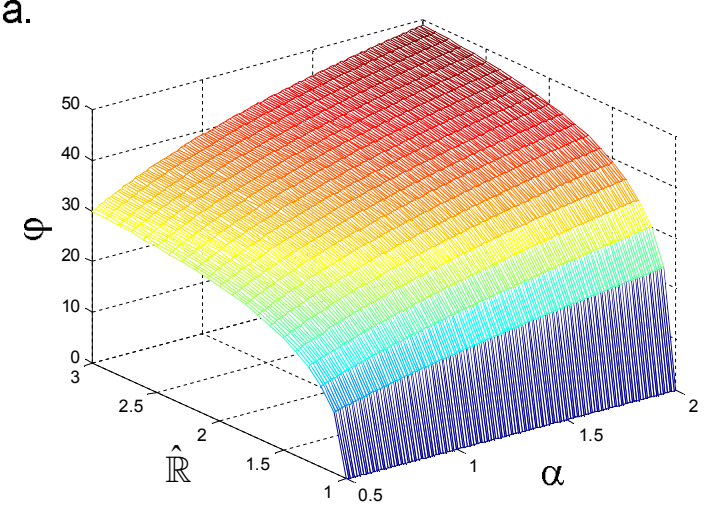

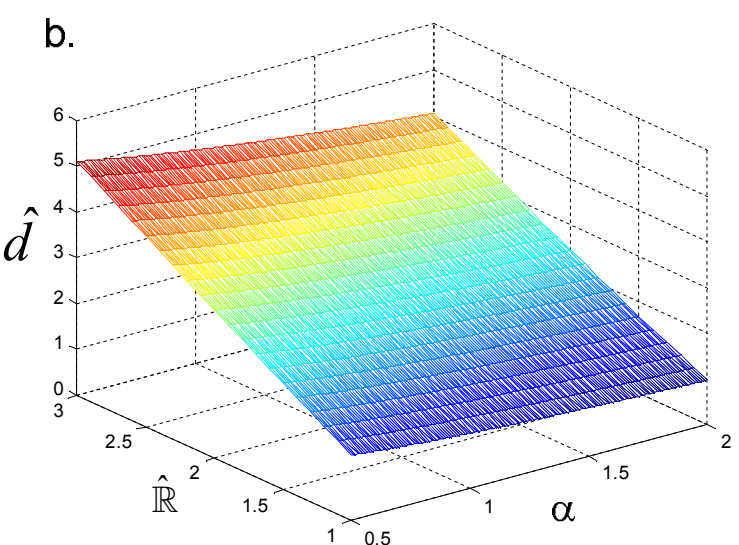

Fig. 5: Representation of relationship between $\varphi, \alpha$ and $\hat{\mathbb{R}}$ such that the dynamic stiffness at the static equilibrium position is equal to zero.

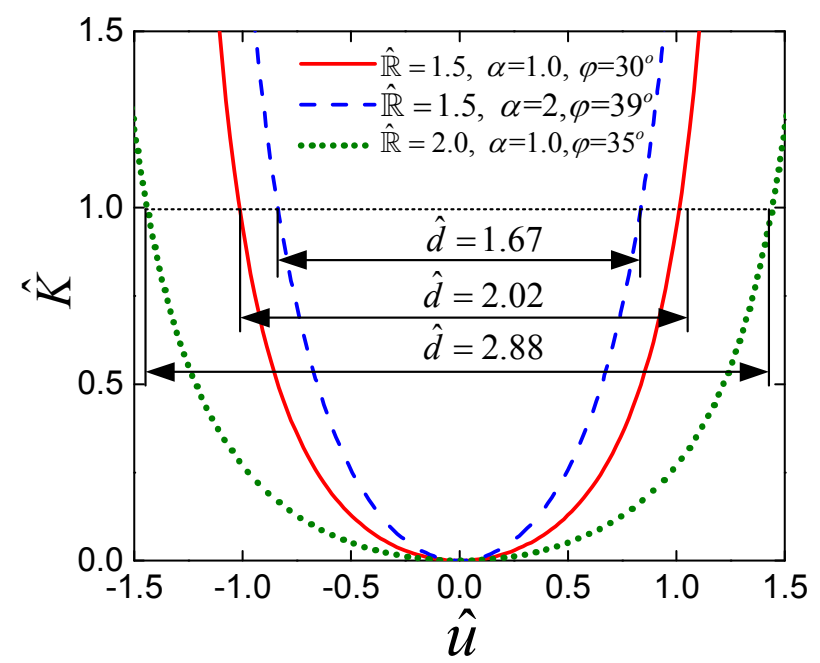

Fig. 6: Dynamic stiffness curve having $\hat{K}_{S E P}=0$. 


\section{Virtual modeling of the seat suspension system}

Fig. 7 shows virtual prototyping model of the seat suspension system created by using the SOLIDWORKS software. This model includes the components which have the shape and dimension of the physical model and it contains information about the mass and inertia properties of these components. Besides, the geometric constrains showing the characteristic of the seat suspension system is employed to build the virtual model. Then, by applying dynamic analysis in the COSMOSMotion module of the Solidworks product family, the virtual model of seat suspension system is tested and evaluated the real behaviour through harmonic excitations form the base. The parameter values used in the simulation are listed in table 1. For these parameters, the system achieves the desirable static equilibrium position.

Table 1: simulation parameter

\begin{tabular}{|l|l|}
\hline Parameters & Values \\
\hline Stiffness of load support spring $\left(\mathrm{K}_{1}\right)$ & $17 \mathrm{~N} / \mathrm{mm}$ \\
\hline Radius of the wedge-cam $(\mathrm{R})$ & $60 \mathrm{~mm}$ \\
\hline Radius of the roller $(\mathrm{r})$ & $20 \mathrm{~mm}$ \\
\hline The angle of the wedge-cam $(\varphi)$ & $37^{\circ}$ \\
\hline Damping ratio & 0.06 \\
\hline Weight & $57 \mathrm{Kg}$ \\
$\quad+$ Part 15 and 9 & $10 \mathrm{Kg}$ \\
$\quad+$ Isolated object & $47 \mathrm{Kg}$ \\
\hline Initial length of spring $1\left(L_{o l}\right)$ & $175 \mathrm{~mm}$ \\
\hline Initial length of spring $2\left(L_{o 2}\right)$ & $80 \mathrm{~mm}$ \\
\hline Time step & $0.01 \mathrm{~s}$ \\
\hline Size of seat frame & $300 \times 400 \mathrm{~mm}$ \\
\hline
\end{tabular}

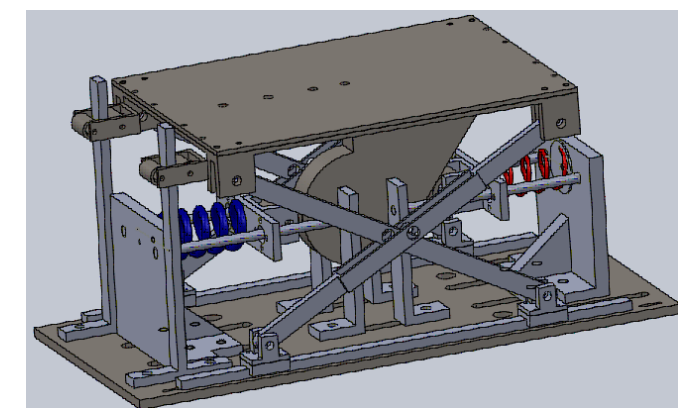

Fig. 7: Show of the virtual prototyping model of seat suspension system.

\section{Simulation results}

\subsection{Comparison of isolation performance between the proposed and traditional model}

In this section, the sinusoidal excitations with the amplitude of $10 \mathrm{~mm}$ and frequency changing from 0.5 to $5 \mathrm{~Hz}$ are employed to assess the isolation effectiveness of the proposed system. The parameters of the system are set as following: the spring ratio $\alpha=1.7$ and $\hat{\mathbb{R}}=1.37$. For which, the dimensionless dynamic stiffness of the proposed model at the static equilibrium position achieves the value of 0.1 . The frequency performance of the proposed model compared with that of the traditional system in which the AM is not considered (called model without AM) is shown in Fig. 8. Here, the blue solid line denotes the response of the seat suspension system using the AM, the red dashed line portraits for the model without AM. It can be seen that, the model with AM can isolate for larger excitation frequencies than $0.5 \mathrm{~Hz}$, whiles, the system without AM occurs resonant phenomenon around $2 \mathrm{~Hz}$ and only offers isolation effectiveness when the frequency is bigger than $3 \mathrm{~Hz}$. Furthermore, the vibration attenuation of the traditional system is lower than the proposed system. 


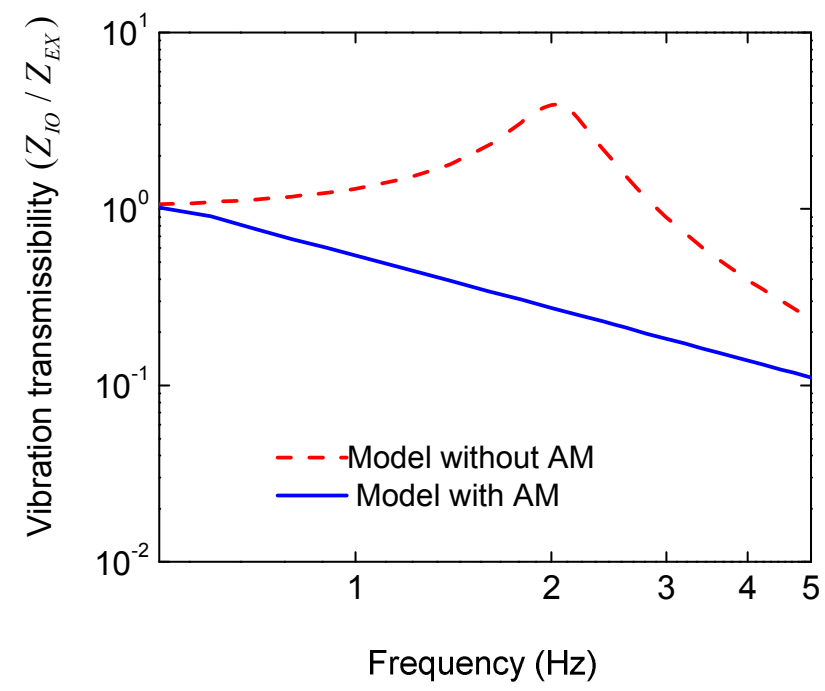

Fig. 8: Comparison of the isolation effectiveness between the proposed (blue solid line) and traditional (red dashed line) models. Specifically, $Z_{I O}$ and $Z_{E X}$ are the absolute displacement amplitude of the isolated object at the steady state and the amplitude of the excitation signal, respectively.

a.

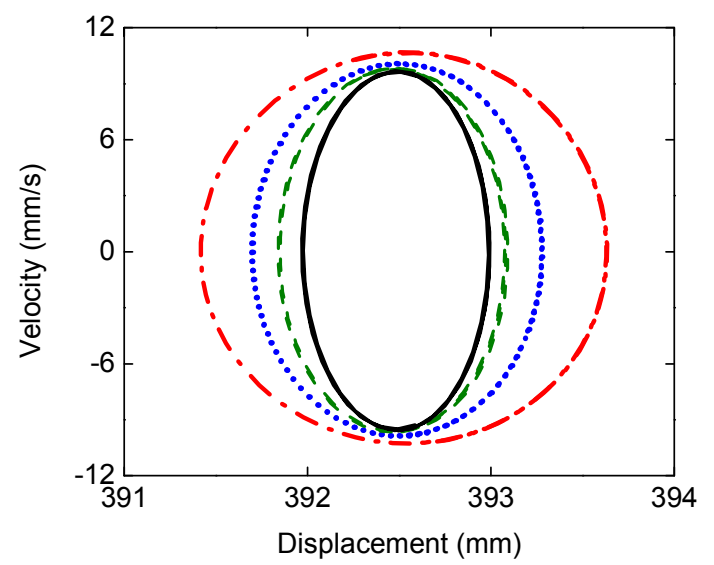

b

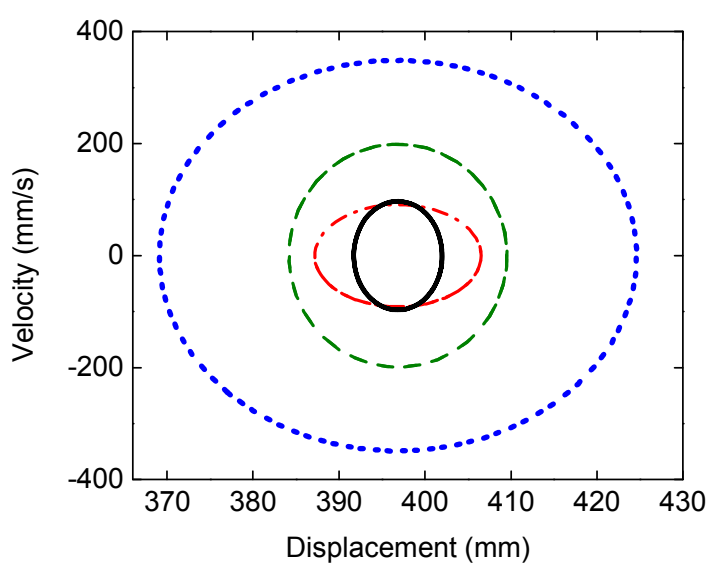

Fig. 9: Phase trajectories of seat suspension system under excitation amplitude $10 \mathrm{~mm}$ and different frequencies including $1.5 \mathrm{~Hz}$ (red dashed dot line); $2 \mathrm{~Hz}$ (blue dot line); $2.5 \mathrm{~Hz}$ (olive dashed line); and 3Hz (black solid line): (a) model with AM;

(b) model without AM.

In addition, the phase orbits of the system are also plotted in Fig. 9 which is a two-dimensional plot of displacement and velocity of the isolated object. In this case, the excitation amplitude $=10 \mathrm{~mm}$ and frequency $=1.5 ; 2.0$; 2.5 and $3.0 \mathrm{~Hz}$ are used. It can be observed that the behavior of the proposed and traditional model is to form a circular trajectory for each excitation frequency. This steady state response is like harmonic motion. The size of ellipse is related to the excitation frequency. As the frequency is increased from 1.5 to $3 \mathrm{~Hz}$, with the proposed model, the size of ellipse is shrunk. This means that the velocity and displacement of the isolated mass are reduced. This result also confirmed that the proposed system offers a good ability for preventing the transmissibility of energy from the base to the mass under the low frequency excitations. Oppositely, with model without AM, the size of the ellipse is increased as the excitation frequency increases from 1.5 to $2.5 \mathrm{~Hz}$.

\subsection{Effect of $K_{\text {SEP }}$ on dynamic behavior}

In this case, the dynamic stiffness at the static equilibrium position is changed by adjusting the spring ratio $\alpha$. Other parameters of the model and the excitation condition are not changed. Specifically, the values of $\hat{K}_{S E P}$ are equal to $0.16,0.1 ; 0.02$ corresponding to the ratio of spring $\alpha=1.5,1.7$ and 2.0 , respectively. The phase trajectory is presented in Fig.10. Here, the black dot line denotes the phase path of system with $\hat{K}_{S E P}=0.16$, the blue dashed line presents for $\hat{K}_{S E P}=0.1$ and the red solid line draws for $\hat{K}_{S E P}=0.02$. The result reveals that the steady state response of system is 
periodic and the size of the phase orbit is extend as the value of $K_{S E P}$ is increased.

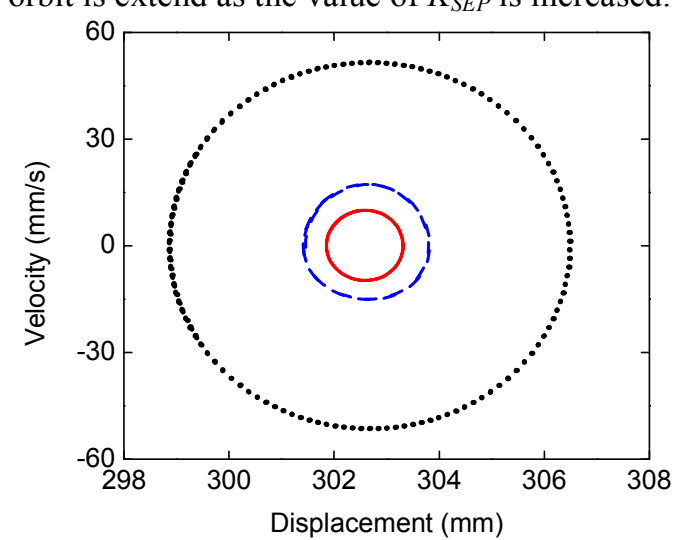

Fig. 10: Phase orbit of the proposed model with three different values of $\mathrm{K}_{\mathrm{SEP}}$ including 0.16 (black dot line); 0.1 (blue dashed line); and 0.02 (red solid line).

Besides, the benefit of low dynamic stiffness value at the equilibrium position is also expressed in Fig. 11. When the $K_{S E P}$ is reduced, the acceleration response of the proposed model is also reduced. This is one of important merits of the proposed system to improve ride comfort for driver and passengers.

a.

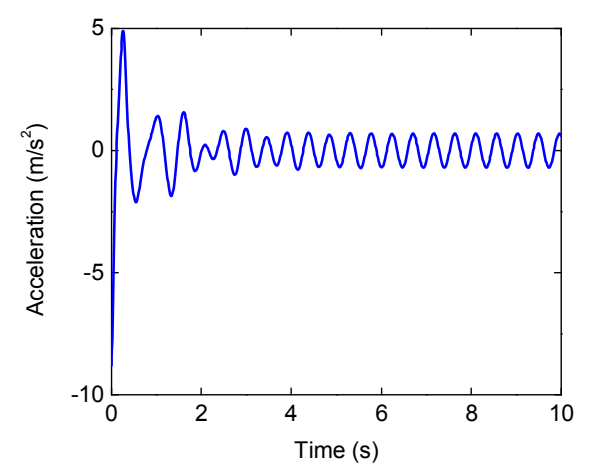

b.

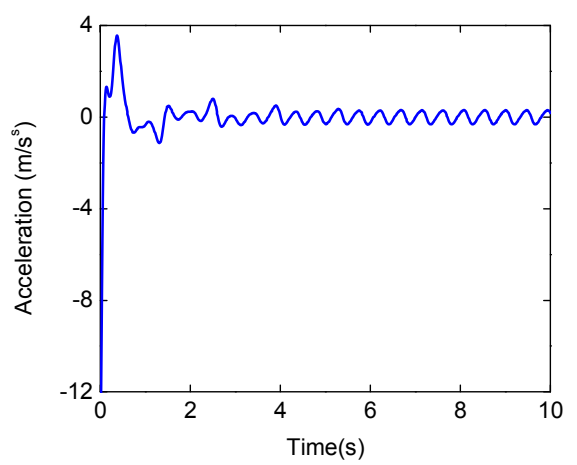

C.

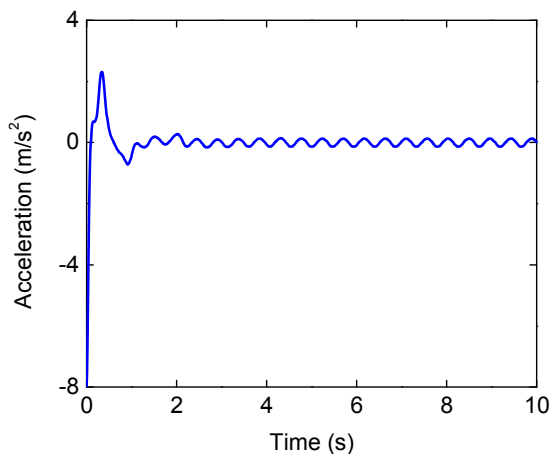

Fig. 11: Time history of acceleration for three different values of $\mathrm{K}_{\mathrm{SEP}}$ : (a) $\mathrm{K}_{\mathrm{SEP}}=0.16$; (b) $\mathrm{K}_{\mathrm{SEP}}=0.1$; and (c) $\mathrm{K}_{\mathrm{SEP}}=0.02$.

\subsection{Effect of slope of the dynamic stiffness curve on the isolation response}

In order to realize this work, the parameters of model with AM are considered in two cases as following: first case: $\varphi=37^{\circ}, \alpha=1.98$, and $\hat{\mathbb{R}}=1.4$; second one: $\varphi=37^{\circ}, \alpha=1.7$, and $\hat{\mathbb{R}}=1.5$. For which, the value of $K_{\text {SEP }}$ is always equal to zero but the first case has smaller distance $d$ than the second case. Specifically, by using Eq. (15) the distance $d$ is calculated as following: first case $\hat{d}_{1}=1.53$ and second case $\hat{d}_{2}=1.75$. The dynamic simulation of the system is shown in Fig. 12(a). The both of two cases, the amplitude and frequency of excitation are $55 \mathrm{~mm}$ and $2.14 \mathrm{~Hz}$, respectively. It is observed that although $\hat{K}_{S E P}=0$ but the isolation effectiveness is different. The second case offers better isolation effectiveness than the first case. This confirmed by the phase orbit as shown in Fig. 12(b). 
a.

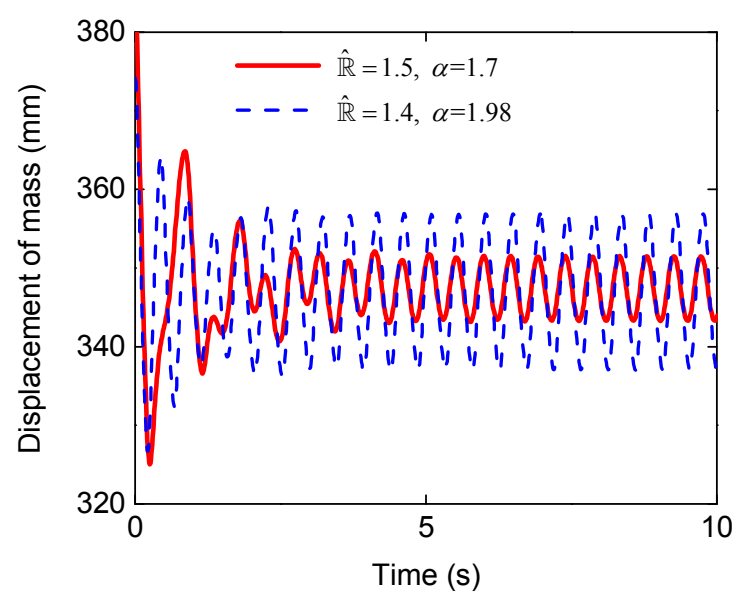

b.

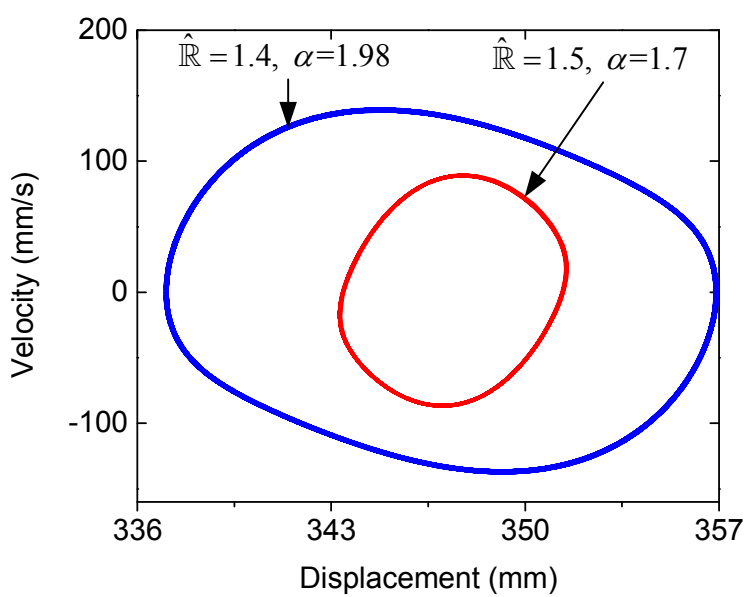

Fig. 12: Comparison of the isolation response of the model with AM for two cases comprising case one: $\varphi=37^{\circ}, \alpha=1.98$, and

$\hat{\mathbb{R}}=1.4$ (denoted by blue line) and case two: $\varphi=37^{\circ}, \alpha=1.7$, and $\hat{\mathbb{R}}=1.5$ (drawn by red line): (a) time history of displacement; (b) phase path.

\section{Conclusion}

An innovative seat suspension system for vehicle has been introduced and analyzed. The dichotomy between low stiffness and load bearing capacity had been overcame. The relationship between the parameters of model and the dynamic stiffness had been investigated. By virtual simulation technology, the isolation effectiveness and effects of the dynamic stiffness at the static equilibrium position and the slope of the stiffness curve on dynamic response of the system has been analyzed. Besides, a comparison of the isolation performance between the model with AM and model without AM was realized. The simulation results shown that the innovative seat suspension system can offer a good effectiveness of isolation for the low excitation frequencies. Especially, this result indicates that the suspension system having quasi-zero stiffness at the static equilibrium position and lower slope of the stiffness curve is easier for preventing the kinetic energy from the base to the isolated object.

\section{Acknowledgement}

This research is funded by Vietnam National University HoChiMinh City (VNU-HCM) under grant number C2015-20-04.

\section{References}

Carrella, A., Brennan, M. J. and Waters, T. P., Static analysis of a passive vibration isolation with quasi zero-stiffness characteristic, Journal of sound and vibration, Vol.301, No.3-5 (2007), pp.678 - 689.

Carrella, A., Brennan, M. J., Waters, T. P. and Shin, K., On the design of high-static-low-dynamic stiffness isolator using linear mechanical spring and magnetic, Journal of sound and vibration, Vol.315, No.3 (2008), pp.712-720.

Carrella, A., Brennan, M. J., Waters, T. P. and Jr Lopes, V., Force and displacement transmissibility of a nonlinear isolator with high-static-low-dynamic stiffness. International Journal of Mechanical science, Vol.55, No.1 (2012), pp. 22-29.

Chnin, E. J., Lee, K. T., Winterflood, J., Ju, L. and Blair, D. G., Low frequency vertical geometric anti-spring vibration isolators, Physics letters A, Vol.336, No. 2-3 (2005), pp. 97 - 105.

Graham Kelly, S., Fundamentals of mechanical vibrations, Second edition (2002), McCraw-Hill.

Hao, Z. and Cao, Q., The isolation characteristics of an archetypal dynamical model with stable-quasi-zero-stiffness, Journal of sound and vibration, Vol.340, (2015), pp. 61-79.

Kovacic, I., Brennan, M. J. and Waters, T. P., A study of a nonlinear vibration isolator with a quasi-zero stiffness characteristic, Journal of Sound and Vibration, Vol. 315, No.3 (2008), pp.700-711.

Lee, C. M., Goverdovskiy, V. N. and Temnikov, A. I., Design of springs with "negative" stiffness to improve vehicle driver vibration isolation, Journal of sound and vibration, Vol.302, No.4-5 (2007), pp. 865 - 874.

Nakamura, Y., Goto. S. and Wakui, S., Tuning method of a smith predictor for pneumatic active anti-vibration apparatuses, Journal of Advanced Mechanical Design, Systems, Manufacturing, Vol.7, No.4 (2013), pp. 666-576. 
Paddan, G. S. and Griffin, M. J., Evaluation of whole-body vibration in vehicle, Journal of Sound and Vibration, Vol.253, No.1 (2002), pp.195 - 213.

Plaut, R. H., Favor, H. M., Jeffers, A. E. and Virgin, L. N., Vibration isolation using buckled or pre-bent columns-part 1: Two-dimensional motion of horizontal rigid bar, Journal of Sound and Vibration, Vol.310, No.1-2 (2008), pp. $409-420$.

Santillan, S., Virgin, L. N. and Plaut, R. H., Equilibria and vibration of a heavy pinched loop, Journal of Sound and Vibration, Vol.288, No.1-2 (2005), pp.81 - 90.

Shirani, H, Nakamura, Y. and Wakui, S., Flow disturbance composition based on off-line estimated signal for a pneumatic isolation table, Journal of Advanced Mechanical Design, Systems, Manufacturing, Vol.7, No.4 (2013), pp. 498-505.

Thorby, D., Structural dynamic and vibration in practice, First edition (2008), Elsevier Ltd.

Virgin, L. N., Santillan, S. T. and Plaut R. H., Vibration isolation using extreme geometric nonlinearity, Journal of Sound and Vibration, Vol. 315, No.3 (2008), pp.721 - 731.

Virgin, L. N. and Davis R. B., Vibration isolation using buckled struts, Journal of sound and vibration, Vol.260, No.5 (2003), pp.965 - 973.

Winterflood, J., Barber, T. A. and Blair, D. G., Mathematical analysis of Euler spring vibration isolator, Physics letters A, Vol.300, No.2-3 (2002), pp.131 - 139. 